(C) 2005 American Chemical Society, Organometallics, Tanaka om058053i Supporting Info Page 1

Supporting Information

\title{
Unpredicted Cyclization of Enyne Having a Keto-Carbonyl \\ Group on Alkyne Using a Ruthenium Catalyst Under
}

\author{
Ethylene Gas
}

\author{
Daisuke Tanaka, ${ }^{a}$ Yoshihiro Sato*a and Miwako Mori*b \\ ${ }^{a}$ Graduate School of Pharmaceutical Sciences, Hokkaido University, \\ Sapporo 060-0812, Japan, 'Health Sciences University of Hokkaido,
}

Toubetsu, Hokkaido 061-0293, Japan

General procedure and the spectral data

S2-S23 
(C) 2005 American Chemical Society, Organometallics, Tanaka om058053i Supporting Info Page 2

General Procedure. General Information. ${ }^{1} \mathrm{H}$ NMR and ${ }^{13} \mathrm{C}$ NMR were recorded on a JEOL EX-270 (270 MHz for ${ }^{1} \mathrm{H}, 67.5 \mathrm{MHz}$ for $\left.{ }^{13} \mathrm{C}\right)$, or JEOL AL-400 (400 MHz for ${ }^{1} \mathrm{H}, 100 \mathrm{MHz}$, for ${ }^{13} \mathrm{C}$ ) instrument in $\mathrm{CDCl}_{3}$ with tetramethylsilane as an internal standard otherwise mentioned. Data are reported as follows: chemical shift in ppm, multiplicity $(\mathrm{s}=$ singlet, $\mathrm{d}=$ doublet, $\mathrm{t}=$ triplet, $\mathrm{q}=$ quartet, $\mathrm{m}=$ multiplet, $\mathrm{br}=$ broad signal), coupling constant $(\mathrm{Hz})$, integration. Infrared spectra (IR) were obtained on a Perkin Elmer 1605 FTIR spectrometer. Mass spectra were obtained on either a JEOL JMS-FABmate (EI), a JEOL JMS-HX110 (FAB), or a JEOL JMS-700TZ (ESI). Silica gel column chromatography was performed by Merck Silica Gel 60 (70-230 or $230-400$ mesh ATM). For analytical or preparative TLC, Merck Silica Gel $60 \mathrm{PF}_{254}$ was used.

Toluene was distilled under an argon atmosphere from $\mathrm{Na}$ /benzophenone. All the solvents for the reaction were degassed through a freeze-pump-thaw cycle. Ethylene gas was purified by passing through the aqueous $\mathrm{CuCl}$ solution ( $2 \mathrm{~g}$ of $\mathrm{CuCl}$ in $180 \mathrm{~mL}$ of saturated aqueous $\left.\mathrm{NH}_{4} \mathrm{Cl}\right)$, concentrated 
$\mathrm{H}_{2} \mathrm{SO}_{4}$, and a $\mathrm{KOH}$ tube.

General Procedure for Cyclization of Enyne: A toluene solution of enyne and $5 \mathrm{~mol} \%$ of $\mathrm{Cp}^{*} \mathrm{RuCl}(\mathrm{cod})$ was stirred at room temperature under ethylene gas $(1 \mathrm{~atm})$. After the spot of the starting material was disappeared on TLC, the solevent was removed and the residue was purified by column chromatography on silica gel.

\section{1-[1-(4,4-Bisbenzyloxymethyl-2-methylcyclopent-1-enyl)cyclopropyl]et}

hanone (3a)

According to the general procedure, a solution of $1 \mathrm{a}(56.5 \mathrm{mg}, 0.15 \mathrm{mmol})$

and $\mathrm{Cp} * \mathrm{RuCl}(\mathrm{cod})(2.8 \mathrm{mg}, 5 \mathrm{~mol} \%)$ in toluene $(5.0 \mathrm{~mL}, 0.03 \mathrm{M})$ was stirred at room temperature for $3 \mathrm{~h}$ under an ethylene. The crude residue was purified by column chromatography on silica gel (benzene) to yield $\mathbf{4 a}$ (60.6 mg, quant.) as a colorless oil. IR (neat) $1692,1453 \mathrm{~cm}^{-1} ;{ }^{1} \mathrm{H}$ NMR $\left(270 \mathrm{MHz}, \mathrm{CDCl}_{3}\right) \delta 0.81(\mathrm{dd}, J=3.6,7.2 \mathrm{~Hz}, 2 \mathrm{H}), 1.35(\mathrm{dd}, J=3.6,7.2$ 
(C) 2005 American Chemical Society, Organometallics, Tanaka om058053i Supporting Info Page 4

$\mathrm{Hz}, 2 \mathrm{H}), 1.63(\mathrm{~s}, 3 \mathrm{H}), 2.07(\mathrm{~s}, 3 \mathrm{H}), 2.25(\mathrm{~s}, 2 \mathrm{H}), 2.28(\mathrm{~s}, 2 \mathrm{H}), 3.42(\mathrm{~s}, 4$

$\mathrm{H}), 4.51(\mathrm{q}, J=12.4,15.6 \mathrm{~Hz}, 4 \mathrm{H}), 7.24-7.34(\mathrm{~m}, 10 \mathrm{H}) ;{ }^{13} \mathrm{C}$ NMR $(100$

$\left.\mathrm{MHz}, \mathrm{CDCl}_{3}\right) \delta 14.7,19.1,28.4,30.4,43.0,45.0,73.2,74.1,127.3,127.4$,

128.2, 131.8, 137.0, 138.6, 209.5; LRMS (El) $\mathrm{m} / \mathrm{z} 404\left(\mathrm{M}^{+}\right), 313,296,283$,

91; HRMS (EI) calcd for $\mathrm{C}_{27} \mathrm{H}_{32} \mathrm{O}_{3}$ 404.2351, found 404.2345; Anal. Calcd

for $\mathrm{C}_{27} \mathrm{H}_{32} \mathrm{O}_{3}: \mathrm{C}, 80.16 ; \mathrm{H}, 7.97$. Found: $\mathrm{C}, 80.21 ; \mathrm{H}, 8.17$.

1-[1-(3,8,8-Trimethyl-7,9-dioxaspiro[4.5]dec-2-en-2-yl)cyclopropyl]etha

none (3b)

Mp. 63-65 ${ }^{\circ} \mathrm{C}$; IR $\left(\mathrm{CHCl}_{3}\right) 1688,1148 \mathrm{~cm}^{-1} ;{ }^{1} \mathrm{H}$ NMR $\left(270 \mathrm{MHz}, \mathrm{CDCl}_{3}\right) \delta$

$0.86(\mathrm{~d}, J=2.4 \mathrm{~Hz}, 1 \mathrm{H}), 0.87(\mathrm{~d}, J=2.4 \mathrm{~Hz}, 1 \mathrm{H}), 1.39(\mathrm{~d}, J=2.2 \mathrm{~Hz}, 1 \mathrm{H})$,

$1.41(\mathrm{~d}, J=2.2 \mathrm{~Hz}, 1 \mathrm{H}), 1.43(\mathrm{~s}, 3 \mathrm{H}), 1.44(\mathrm{~s}, 3 \mathrm{H}), 1.68(\mathrm{~s}, 3 \mathrm{H}), 2.14(\mathrm{~s}$,

$3 \mathrm{H}), 2.25(\mathrm{~s}, 2 \mathrm{H}), 2.34(\mathrm{q}, J=1.4 \mathrm{~Hz}, 2 \mathrm{H}), 3.66(\mathrm{~d}, J=7.3 \mathrm{~Hz}, 2 \mathrm{H}), 3.69$

$(\mathrm{d}, J=7.3 \mathrm{~Hz}, 2 \mathrm{H}) ;{ }^{13} \mathrm{C}$ NMR $\left(100 \mathrm{MHz}, \mathrm{CDCl}_{3}\right) \delta 14.9,19.1,23.6,24.5$,

$28.3,30.6,39.1,44.1,45.7,69.3,97.7,131.8,136.7,208.4 ;$ LRMS (EI) $\mathrm{m} / \mathrm{z}$

$264\left(\mathrm{M}^{+}\right), 249,206,176,161,133,43$; HRMS (EI) calcd for $\mathrm{C}_{16} \mathrm{H}_{24} \mathrm{O}_{3}$ 
(C) 2005 American Chemical Society, Organometallics, Tanaka om058053i Supporting Info Page 5

264.17253, found 264.17168; Anal. Calcd for $\mathrm{C}_{16} \mathrm{H}_{24} \mathrm{O}_{3}: \mathrm{C}, 72.69 ; \mathrm{H}, 9.15$.

Found: C, 72.61; H, 9.28.

1-[1-(3,8,8-Trimethyl-7,9-dioxaspiro[4.5]dec-2-en-2-yl)cyclopropyl]but anone (3c)

IR (neat) $1693,1124 \mathrm{~cm}^{-1} ;{ }^{1} \mathrm{H}$ NMR $\left(400 \mathrm{MHz}, \mathrm{CDCl}_{3}\right) \delta 0.82(\mathrm{~d}, J=3.2$ $\mathrm{Hz}, 1 \mathrm{H}), 0.83(\mathrm{~d}, J=3.2 \mathrm{~Hz}, 1 \mathrm{H}), 0.87(\mathrm{t}, J=7.2 \mathrm{~Hz}, 3 \mathrm{H}), 1.37(\mathrm{~d}, J=$ $3.2 \mathrm{~Hz}, 1 \mathrm{H}), 1.39(\mathrm{~d}, J=3.2 \mathrm{~Hz}, 1 \mathrm{H}), 1.44(\mathrm{~s}, 3 \mathrm{H}), 1.44(\mathrm{~s}, 3 \mathrm{H}), 1.53(\mathrm{tq}$, $J=7.2,7.2 \mathrm{~Hz}, 2 \mathrm{H}), 1.67(\mathrm{~s}, 3 \mathrm{H}), 2.26(\mathrm{~s}, 2 \mathrm{H}), 2.32(\mathrm{~s}, 2 \mathrm{H}), 2.43(\mathrm{t}, J=$

$7.2 \mathrm{~Hz}, 2 \mathrm{H}), 3.66(\mathrm{~d}, J=12.0 \mathrm{~Hz}, 2 \mathrm{H}), 3.69(\mathrm{~d}, J=12.0 \mathrm{~Hz}, 2 \mathrm{H}) ;{ }^{13} \mathrm{C}$ $\operatorname{NMR}\left(100 \mathrm{MHz}, \mathrm{CDCl}_{3}\right) \delta 14.0,15.0,17.4,18.9,23.7,24.3,30.4,39.2$, 42.3, 44.2, 45.7, 69.4, 97.7, 131.6, 136.7, 210.1; LRMS (EI) $\mathrm{m} / \mathrm{z} 292\left(\mathrm{M}^{+}\right)$, $277,263,234,219,204,71,43 ; \mathrm{HRMS}(\mathrm{EI})$ calcd for $\mathrm{C}_{18} \mathrm{H}_{28} \mathrm{O}_{3} 292.20383$, found 292.20361; Anal. Calcd for $\mathrm{C}_{18} \mathrm{H}_{28} \mathrm{O}_{3}$ : C, 73.93; H, 9.65. Found: $\mathrm{C}$, 73.92; H, 9.70 . 
(C) 2005 American Chemical Society, Organometallics, Tanaka om058053i Supporting Info Page 6

2-Methyl-1-[1-(3,8,8-trimethyl-7,9-dioxaspiro[4.5]dec-2-en-2-yl)cyclopr opyl]propanone (3d)

IR (neat) $1691,1156 \mathrm{~cm}^{-1} ;{ }^{1} \mathrm{H} \mathrm{NMR}\left(400 \mathrm{MHz}, \mathrm{CDCl}_{3}\right) \delta 0.84(\mathrm{~d}, J=3.6$ $\mathrm{Hz}, 1 \mathrm{H}), 0.86(\mathrm{~d}, J=3.6 \mathrm{~Hz}, 1 \mathrm{H}), 1.01(\mathrm{~d}, J=6.8 \mathrm{~Hz}, 6 \mathrm{H}), 1.36(\mathrm{~d}, J=$ $3.6 \mathrm{~Hz}, 1 \mathrm{H}), 1.38(\mathrm{~d}, J=3.6 \mathrm{~Hz}, 1 \mathrm{H}), 1.44(\mathrm{~s}, 6 \mathrm{H}), 1.66(\mathrm{~s}, 3 \mathrm{H}), 2.26(\mathrm{~s}$, $2 \mathrm{H}), 2.33(\mathrm{~s}, 2 \mathrm{H}), 2.95$ (septetd, $J=6.8 \mathrm{~Hz}, 1 \mathrm{H}), 3.66(\mathrm{~d}, J=11.2 \mathrm{~Hz}, 2$ H), $3.69(\mathrm{~d}, J=11.2 \mathrm{~Hz}, 2 \mathrm{H}) ;{ }^{13} \mathrm{C} \operatorname{NMR}\left(100 \mathrm{MHz}, \mathrm{CDCl}_{3}\right) \delta 15.2,18.7$, $19.4,23.8,24.2,29.7,36.8,39.2,44.3,45.8,69.3,97.7,131.5,136.6$, 214.5; LRMS (EI) m/z $292\left(\mathrm{M}^{+}\right), 277,263,219,204,189,164$; HRMS (EI) calcd for $\mathrm{C}_{18} \mathrm{H}_{28} \mathrm{O}_{3}$ 292.20383, found 292.20408; Anal. Calcd for $\mathrm{C}_{18} \mathrm{H}_{28} \mathrm{O}_{3}$ : C, 73.93; H, 9.65. Found: C, 73.85; H, 9.69.

1-Cyclohexyl-1-[1-(3,8,8-trimethyl-7,9-dioxaspiro[4.5]dec-2-en-2-yl)cycl opropyl]methanone (3e)

IR (neat) $1686,1155 \mathrm{~cm}^{-1} ;{ }^{1} \mathrm{H}$ NMR $\left(400 \mathrm{MHz}, \mathrm{CDCl}_{3}\right) \delta 0.81(\mathrm{~d}, J=3.2$ $\mathrm{Hz}, 1 \mathrm{H}), 0.83(\mathrm{~d}, J=3.2 \mathrm{~Hz}, 1 \mathrm{H}), 1.17-1.36(\mathrm{~m}, 6 \mathrm{H}), 1.34(\mathrm{~d}, J=3.2 \mathrm{~Hz}$, 
(C) 2005 American Chemical Society, Organometallics, Tanaka om058053i Supporting Info Page 7

$1 \mathrm{H}), 1.36(\mathrm{~d}, J=3.2 \mathrm{~Hz}, 1 \mathrm{H}), 1.44(\mathrm{~s}, 6 \mathrm{H}), 1.65(\mathrm{~s}, 3 \mathrm{H}), 1.70-1.77(\mathrm{~m}, 4$

H), 2.28-2.30 (m, $4 \mathrm{H}), 2.68(\mathrm{tt}, J=3.2,11.2 \mathrm{~Hz}, 1 \mathrm{H}), 3.67(\mathrm{~d}, J=11.2 \mathrm{~Hz}$, $2 \mathrm{H}), 3.70(\mathrm{~d}, J=11.2 \mathrm{~Hz}, 2 \mathrm{H}) ;{ }^{13} \mathrm{C} \mathrm{NMR}\left(100 \mathrm{MHz}, \mathrm{CDCl}_{3}\right) \delta 15.1,18.4$, $23.6,24.4,25.9,26.0,29.4,29.7,39.1,44.1,45.9,47.5,69.4,97.7,131.4$, 136.7, 213.6; LRMS (EI) $m / z 332\left(\mathrm{M}^{+}\right), 317,303,274,259,244,204,83$;

HRMS (EI) calcd for $\mathrm{C}_{21} \mathrm{H}_{32} \mathrm{O}_{3} 332.23513$, found 332.23450; Anal. Calcd for $\mathrm{C}_{21} \mathrm{H}_{32} \mathrm{O}_{3}$ : C, 75.86; $\mathrm{H}, 9.70$. Found: C, 75.90; H, 9.52.

\section{4-Phenyl-1-[1-(3,8,8-trimethyl-7,9-dioxaspiro[4.5]dec-2-en-2-yl)cyclopr} opyl]buthanone (3f)

IR (neat) $1692,1155 \mathrm{~cm}^{-1} ;{ }^{1} \mathrm{H}$ NMR $\left(400 \mathrm{MHz}, \mathrm{CDCl}_{3}\right) \delta 0.81$ (dd, $J=3.2$, $6.8 \mathrm{~Hz}, 2 \mathrm{H}), 1.37(\mathrm{dd}, J=3.2,6.8 \mathrm{~Hz}, 2 \mathrm{H}), 1.43(\mathrm{~s}, 3 \mathrm{H}), 1.44(\mathrm{~s}, 3 \mathrm{H})$, $1.60(\mathrm{~s}, 3 \mathrm{H}), 1.84(\mathrm{tt}, J=7.2,7.2 \mathrm{~Hz}, 2 \mathrm{H}), 2.21(\mathrm{~s}, 2 \mathrm{H}), 2.23(\mathrm{~s}, 2 \mathrm{H})$, $2.45(\mathrm{t}, J=7.2 \mathrm{~Hz}, 2 \mathrm{H}), 2.58(\mathrm{t}, J=7.2 \mathrm{~Hz}, 2 \mathrm{H}), 3.60(\mathrm{~d}, J=11.2 \mathrm{~Hz}, 2$ H), $3.63(\mathrm{~d}, J=11.2 \mathrm{~Hz}, 2 \mathrm{H}), 7.13-7.29(\mathrm{~m}, 5 \mathrm{H}) ;{ }^{13} \mathrm{C}$ NMR $(100 \mathrm{MHz}$, $\left.\mathrm{CDCl}_{3}\right) \delta 14.9,19.0,23.9,24.1,25.3,30.3,35.1,39.1,39.4,44.1,45.7$, 
(C) 2005 American Chemical Society, Organometallics, Tanaka om058053i Supporting Info Page 8

69.3, 97.6, 125.6, 128.1, 128.3, 131.3, 136.8, 141.4, 209.7; LRMS (EI) $\mathrm{m} / \mathrm{z}$

$368\left(\mathrm{M}^{+}\right), 353,310,277,219,189,105,91,43$; HRMS (EI) calcd for

$\mathrm{C}_{24} \mathrm{H}_{32} \mathrm{O}_{3} 368.2351$, found 368.2349; Anal. Calcd for $\mathrm{C}_{24} \mathrm{H}_{32} \mathrm{O}_{3}: \mathrm{C}, 78.22 ; \mathrm{H}$,

8.75. Found: $\mathrm{C}, 77.95 ; \mathrm{H}, 8.85$.

\section{4-Benzyloxy-1-[1-(3,8,8-trimethyl-7,9-dioxaspiro[4.5]dec-2-en-2-yl)cycl}

opropyl]buthanone $(3 \mathrm{~g})$

IR (neat) $1693,1155,1102 \mathrm{~cm}^{-1} ;{ }^{\prime} \mathrm{H}$ NMR $\left(270 \mathrm{MHz}, \mathrm{CDCl}_{3}\right) \delta 0.83(\mathrm{~d}, J=$

$3.2 \mathrm{~Hz}, 2 \mathrm{H}), 1.37$ (d, $J=3.2 \mathrm{~Hz}, 2 \mathrm{H}), 1.42(\mathrm{~s}, 3 \mathrm{H}), 1.44(\mathrm{~s}, 3 \mathrm{H}), 1.65(\mathrm{~s}$,

$3 \mathrm{H}), 1.83(\mathrm{tt}, J=6.8,6.8 \mathrm{~Hz}, 2 \mathrm{H}), 2.25(\mathrm{~s}, 2 \mathrm{H}), 2.30(\mathrm{~s}, 2 \mathrm{H}), 2.58(\mathrm{t}, J=$

$6.8 \mathrm{~Hz}, 2 \mathrm{H}), 3.44(\mathrm{t}, J=6.8 \mathrm{~Hz}, 2 \mathrm{H}), 3.63(\mathrm{~d}, J=11.6 \mathrm{~Hz}, 2 \mathrm{H}), 3.67(\mathrm{~d}, J$

$=11.6 \mathrm{~Hz}, 2 \mathrm{H}), 4.46(\mathrm{~s}, 2 \mathrm{H}), 7.26-7.33(\mathrm{~m}, 5 \mathrm{H}) ;{ }^{13} \mathrm{C}$ NMR $(100 \mathrm{MHz}$,

$\left.\mathrm{CDCl}_{3}\right) \delta 14.9,19.0,23.8,24.1,24.3,30.4,36.9,39.1,44.1,45.7,69.3$,

$72.8,97.6,127.3,127.4,128.1,131.4,136.8,138.3,209.6$; LRM (EI) $\mathrm{m} / \mathrm{z}$

$398\left(\mathrm{M}^{+}\right), 383,340,310,292,277,264,219,201,105,91 ; \mathrm{HRMS}(\mathrm{EI})$

calcd for $\mathrm{C}_{25} \mathrm{H}_{34} \mathrm{O}_{4} 398.2457$, found 398.2459; Anal. Calcd for $\mathrm{C}_{25} \mathrm{H}_{34} \mathrm{O}_{4}: \mathrm{C}$, 
(C) 2005 American Chemical Society, Organometallics, Tanaka om058053i Supporting Info Page 9

75.34; H, 8.60. Found: C, 75.17; H, 8.77.

3-[4-Methyl-1-(toluene-4-sulfonyl)pyrrolidin-3-ylidene]pent-4-en-2-one

IR (neat) 1686, 1598, 1348, $1163 \mathrm{~cm}^{-1} ;{ }^{1} \mathrm{H}$ NMR $\left(270 \mathrm{MHz}, \mathrm{CDCl}_{3}\right) \delta 1.12$

$(\mathrm{d}, J=7.0 \mathrm{~Hz}, 3 \mathrm{H}), 2.23(\mathrm{~s}, 3 \mathrm{H}), 2.43(\mathrm{~s}, 3 \mathrm{H}), 2.97-3.11(\mathrm{~m}, 2 \mathrm{H}), 3.21$

$(\mathrm{dd}, J=1.9,9.2 \mathrm{~Hz}, 1 \mathrm{H}), 3.76(\mathrm{~d}, J=17.3 \mathrm{~Hz}, 1 \mathrm{H}), 4.23(\mathrm{~d}, J=17.3 \mathrm{~Hz}, 1$

H), $5.13(\mathrm{~d}, J=17.6 \mathrm{~Hz}, 1 \mathrm{H}), 5.35(\mathrm{~d}, J=10.8 \mathrm{~Hz}, 1 \mathrm{H}), 6.42(\mathrm{dd}, J=10.8$,

$17.6 \mathrm{~Hz}, 1 \mathrm{H}), 7.33(\mathrm{~d}, J=8.1 \mathrm{~Hz}, 2 \mathrm{H}), 7.70(\mathrm{~d}, J=8.1 \mathrm{~Hz}, 2 \mathrm{H}) ;{ }^{13} \mathrm{C}$ NMR

$\left(100 \mathrm{MHz}, \mathrm{CDCl}_{3}\right) \delta 19.2,21.6,30.1,36.8,51.7,53.9,119.1,127.7,129.4$,

131.1, 131.9, 133.0, 143.5, 149.2, 201.0; LRMS (EI) $\mathrm{m} / \mathrm{z} 319\left(\mathrm{M}^{+}\right), 276$,

$164,155,122,91,43 ; \mathrm{HRMS}$ (EI) calcd for $\mathrm{C}_{17} \mathrm{H}_{21} \mathrm{NO}_{3} \mathrm{~S} 319.12419$, found 319.12339.

\section{3-(4-Methyldihydrofuran-3-ylidene)pent-4-en-2-one (2j)}

IR (neat) $1685,1631,1604,1091 \mathrm{~cm}^{-1} ;{ }^{1} \mathrm{H}$ NMR $\left(400 \mathrm{MHz}, \mathrm{CDCl}_{3}\right) \delta 1.16$ 
(C) 2005 American Chemical Society, Organometallics, Tanaka om058053i Supporting Info Page 10

(d, $J=6.8 \mathrm{~Hz}, 3 \mathrm{H}), 2.27(\mathrm{~s}, 3 \mathrm{H}), 3.01(\mathrm{dq}, J=6.0,6.8 \mathrm{~Hz}, 1 \mathrm{H}), 3.66(\mathrm{~d}, J$ $=8.4 \mathrm{~Hz}, 1 \mathrm{H}), 3.84(\mathrm{dd}, J=6.0,8.4 \mathrm{~Hz}, 1 \mathrm{H}), 4.42(\mathrm{~d}, J=17.2 \mathrm{~Hz}, 1 \mathrm{H})$, $4.71(\mathrm{~d}, J=17.2 \mathrm{~Hz}, 1 \mathrm{H}), 5.23(\mathrm{~d}, J=18.0 \mathrm{~Hz}, 1 \mathrm{H}), 5.40(\mathrm{~d}, J=11.2 \mathrm{~Hz}$, $1 \mathrm{H}), 6.54(\mathrm{dd}, J=11.2,18.0 \mathrm{~Hz}, 1 \mathrm{H}) ;{ }^{13} \mathrm{C} \mathrm{NMR}\left(100 \mathrm{MHz}, \mathrm{CDCl}_{3}\right) \delta 18.3$, 30.1, 38.1, 71.5, 74.2, 118.8, 130.8, 132.3, 156.4, 200.6; LRMS (EI) $\mathrm{m} / z$ $166\left(\mathrm{M}^{+}\right), 151,137,123,95,43$; HRMS (EI) calcd for $\mathrm{C}_{10} \mathrm{H}_{14} \mathrm{O}_{2}$ 166.09937, found 166.09847 .

\section{2-(3,8,8-Trimethyl-7,9-dioxaspiro[4.5]dec-2-ylidene)but-3-enal (2k)}

IR (neat) $1682,1120 \mathrm{~cm}^{-1} ;{ }^{1} \mathrm{H}$ NMR $\left(400 \mathrm{MHz}, \mathrm{CDCl}_{3}\right) \delta 1.19(\mathrm{~d}, J=7.2$ $\mathrm{Hz}, 3 \mathrm{H}), 1.26(\mathrm{dd}, J=6.4,13.6 \mathrm{~Hz}, 1 \mathrm{H}), 1.42(\mathrm{~s}, 3 \mathrm{H}), 1.44(\mathrm{~s}, 3 \mathrm{H}), 2.02$ $(\mathrm{dd}, J=8.4,13.6 \mathrm{~Hz}, 1 \mathrm{H}), 2.61(\mathrm{~d}, J=18.0 \mathrm{~Hz}, 1 \mathrm{H}), 3.11(\mathrm{ddq}, J=6.4$, $8.4,7.2 \mathrm{~Hz}, 1 \mathrm{H}), 3.25(\mathrm{~d}, J=18.0 \mathrm{~Hz}, 1 \mathrm{H}), 3.45(\mathrm{dd}, J=1.2,11.2 \mathrm{~Hz}, 1$ H), $3.56(\mathrm{~d}, J=11.2 \mathrm{~Hz}, 1 \mathrm{H}), 3.73(\mathrm{dd}, J=1.2,11.2 \mathrm{~Hz}, 1 \mathrm{H}), 3.80(\mathrm{~d}, J=$ $11.2 \mathrm{~Hz}, 1 \mathrm{H}), 5.35(\mathrm{~d}, J=11.6 \mathrm{~Hz}, 1 \mathrm{H}), 5.79(\mathrm{~d}, J=17.6 \mathrm{~Hz}, 1 \mathrm{H}), 6.36$ $(\mathrm{dd}, J=11.6,17.6 \mathrm{~Hz}, 1 \mathrm{H}), 10.03(\mathrm{~s}, 1 \mathrm{H}) ;{ }^{13} \mathrm{C} \mathrm{NMR}\left(100 \mathrm{MHz}, \mathrm{CDCl}_{3}\right) \delta$ 
(C) 2005 American Chemical Society, Organometallics, Tanaka om058053i Supporting Info Page 11

$21.5,22.5,25.2,36.9,37.9,39.5,41.8,67.7,69.4,97.9,118.3,129.2,130.4$ 169.0, 191.1; LRMS (EI) $\mathrm{m} / 2250\left(\mathrm{M}^{+}\right), 235,192,174,162 ; \mathrm{HRMS}(\mathrm{EI})$ calcd for $\mathrm{C}_{15} \mathrm{H}_{22} \mathrm{O}_{3} 250.1569$, found 250.1560 .

\section{2d-2-Methyl-1-[1-(3,8,8-trimethyl-7,9-dioxaspiro[4.5]dec-2-en-2-yl)cycl}

\section{opropyl]propanone (3d-D)}

IR (neat) $1688,1155 \mathrm{~cm}^{-1} ;{ }^{1} \mathrm{H}$ NMR $\left(400 \mathrm{MHz}, \mathrm{CDCl}_{3}\right) \delta 0.81(\mathrm{~d}, J=3.2$ $\mathrm{Hz}, 1 \mathrm{H}), 0.83(\mathrm{~d}, J=3.2 \mathrm{~Hz}, 1 \mathrm{H}), 0.98(\mathrm{~s}, 6 \mathrm{H}), 1.34(\mathrm{~d}, J=3.2 \mathrm{~Hz}, 1 \mathrm{H})$, $1.36(\mathrm{~d}, J=3.2 \mathrm{~Hz}, 1 \mathrm{H}), 1.41(\mathrm{~s}, 6 \mathrm{H}), 1.64(\mathrm{~s}, 3 \mathrm{H}), 2.24(\mathrm{~s}, 2 \mathrm{H}), 2.30(\mathrm{~s}$, $2 \mathrm{H}), 3.65(\mathrm{~s}, 4 \mathrm{H}), 3.63(\mathrm{~d}, J=11.3 \mathrm{~Hz}, 2 \mathrm{H}), 3.67(\mathrm{~d}, J=11.3 \mathrm{~Hz}, 2 \mathrm{H})$; ${ }^{13} \mathrm{C} \mathrm{NMR}\left(67.5 \mathrm{MHz}, \mathrm{CDCl}_{3}\right) \delta 14.9(\mathrm{~s}), 18.5(\mathrm{~s}), 19.1(\mathrm{~s}), 23.6(\mathrm{~s}), 24.0(\mathrm{~s})$, $29.5(\mathrm{~s}), 36.2(\mathrm{t}), 39.0(\mathrm{~s}), 44.1(\mathrm{~s}), 45.6(\mathrm{~s}), 69.3(\mathrm{~s}), 97.8(\mathrm{~s}), 131.8(\mathrm{~s})$ 136.8 (s), 215.2 (s); LRMS (EI) $m / z 293\left(\mathrm{M}^{+}\right), 278,263,235,205,190$, 161; HRMS (EI) calcd for $\mathrm{C}_{18} \mathrm{H}_{27}{ }^{2} \mathrm{HO}_{3}$ 293.2087, found 293.2094. 
(C) 2005 American Chemical Society, Organometallics, Tanaka om058053i Supporting Info Page 12

\section{opyl]ethanone (3b-D)}

IR (neat) $1688,1146 \mathrm{~cm}^{-1} ;{ }^{1} \mathrm{H}$ NMR $\left(400 \mathrm{MHz}, \mathrm{CDCl}_{3}\right) \delta 0.86(\mathrm{~d}, J=3.2$ $\mathrm{Hz}, 1 \mathrm{H}), 0.88(\mathrm{~d}, J=3.2 \mathrm{~Hz}, 1 \mathrm{H}), 1.40(\mathrm{~d}, J=3.2 \mathrm{~Hz}, 1 \mathrm{H}), 1.41(\mathrm{~d}, J=$

$3.2 \mathrm{~Hz}, 1 \mathrm{H}), 1.44(\mathrm{~s}, 3 \mathrm{H}), 1.44(\mathrm{~s}, 3 \mathrm{H}), 1.66(\mathrm{~s}, 2 \mathrm{H}), 2.14(\mathrm{~s}, 3 \mathrm{H}), 2.25(\mathrm{~s}$,

$2 \mathrm{H}), 2.34(\mathrm{~s}, 2 \mathrm{H}), 3.66(\mathrm{~d}, J=11.2 \mathrm{~Hz}, 2 \mathrm{H}), 3.69(\mathrm{~d}, J=11.2 \mathrm{~Hz}, 2 \mathrm{H})$;

${ }^{13} \mathrm{C}$ NMR $\left(67.5 \mathrm{MHz}, \mathrm{CDCl}_{3}\right) \delta 14.4(\mathrm{t}), 18.9(\mathrm{~s}), 23.4(\mathrm{~s}), 24.3(\mathrm{~s}), 28.2(\mathrm{~s})$,

$30.5(\mathrm{~s}), 39.0(\mathrm{~s}), 44.0(\mathrm{~s}), 45.5(\mathrm{~s}), 69.3(\mathrm{~s}), 97.8(\mathrm{~s}), 132.0(\mathrm{~s}), 136.9(\mathrm{~s})$,

208.9 (s); LRMS (EI) m/z $265\left(\mathrm{M}^{+}\right), 250,207,177,162,134,43$; HRMS

(EI) calcd for $\mathrm{C}_{16} \mathrm{H}_{23}{ }^{2} \mathrm{HO}_{3} 265.1787$, found 265.1787.

\section{3-(3d-3,8,8-Trimethyl-7,9-dioxaspiro[4.5]dec-2-ylidene)pent-4-en-2-one}

(2b-D)

IR (neat) $1698,1421,1115 \mathrm{~cm}^{-1} ;{ }^{1} \mathrm{H}$ NMR $\left(400 \mathrm{MHz}, \mathrm{CDCl}_{3}\right) \delta 1.06$ (s, 3

H), $1.12(\mathrm{~d}, J=13.2 \mathrm{~Hz}, 1 \mathrm{H}), 1.32(\mathrm{~s}, 3 \mathrm{H}), 1.34(\mathrm{~s}, 3 \mathrm{H}), 1.97(\mathrm{~d}, J=13.2$

$\mathrm{Hz}, 1 \mathrm{H}), 2.23(\mathrm{~s}, 3 \mathrm{H}), 2.27(\mathrm{~d}, J=16.8 \mathrm{~Hz}, 1 \mathrm{H}), 2.40(\mathrm{~d}, J=16.8 \mathrm{~Hz}, 1$

H), $3.36(\mathrm{~d}, J=11.2 \mathrm{~Hz}, 1 \mathrm{H}), 3.42(\mathrm{~d}, J=11.2 \mathrm{~Hz}, 1 \mathrm{H}), 3.61(\mathrm{~d}, J=11.2$ 
(C) 2005 American Chemical Society, Organometallics, Tanaka om058053i Supporting Info Page 13

$\mathrm{Hz}, 1 \mathrm{H}), 3.65(\mathrm{~d}, J=11.2 \mathrm{~Hz}, 1 \mathrm{H}), 4.93(\mathrm{~d}, J=18.0 \mathrm{~Hz}, 1 \mathrm{H}), 5.13(\mathrm{~d}, J=$

$11.2 \mathrm{~Hz}, 1 \mathrm{H}), 6.41(\mathrm{dd}, J=11.2,18.0 \mathrm{~Hz}, 1 \mathrm{H}) ;{ }^{13} \mathrm{C}$ NMR $(67.5 \mathrm{MHz}$, $\left.\mathrm{CDCl}_{3}\right) \delta 21.9(\mathrm{~s}), 23.1(\mathrm{~s}), 24.3(\mathrm{~s}), 30.6(\mathrm{~s}), 34.4(\mathrm{t}), 39.1(\mathrm{~s}), 40.2(\mathrm{~s})$, $41.3(\mathrm{~s}), 67.6(\mathrm{~s}), 69.5(\mathrm{~s}), 97.9(\mathrm{~s}), 116.0(\mathrm{~s}), 131.2(\mathrm{~s}), 136.0(\mathrm{~s}), 150.7(\mathrm{~s})$, 205.3 (s); LRMS (EI) $\mathrm{m} / 2265\left(\mathrm{M}^{+}\right), 250,207,177,162,134,43$; HRMS (EI) calcd for $\mathrm{C}_{16} \mathrm{H}_{23}{ }^{2} \mathrm{HO}_{3} 265.1787$, found 265.1785.

\section{1-(3,8,8-Trimethyl-7,9-dioxaspiro[4.5]dec-2-en-2-yl)bicyclo[4.1.0]hepta} n-2-one (5)

A solution of $4(76.2 \mathrm{mg}, 0.26 \mathrm{mmol})$ and $\mathrm{Cp} * \mathrm{RuCl}(\mathrm{cod})(5.0 \mathrm{mg}, 5$ mol \%) in toluene $(8.5 \mathrm{~mL}, 0.03 \mathrm{M})$ was stirred at room temperature for 25 $\mathrm{h}$ under argon gas. The crude residue was purified by column chromatography on silica gel (hexane/AcOEt, 5/1) to yield $5(58.2 \mathrm{mg}$, $76 \%$ ) as a colorless oil. IR (neat) $1688,1155 \mathrm{~cm}^{-1} ;{ }^{1} \mathrm{H}$ NMR (400 MHz, $\left.\mathrm{C}_{6} \mathrm{D}_{6}\right) \delta 0.48(\mathrm{dd}, J=4.8,8.0 \mathrm{~Hz}, 1 \mathrm{H}), 0.68(\mathrm{dd}, J=5.6,5.6 \mathrm{~Hz}, 1 \mathrm{H})$, 0.82-0.91 (m, $2 \mathrm{H}), 0.96-1.00(\mathrm{~m}, 1 \mathrm{H}), 1.13(\mathrm{~s}, 3 \mathrm{H}), 1.15(\mathrm{~s}, 3 \mathrm{H})$, 
(C) 2005 American Chemical Society, Organometallics, Tanaka om058053i Supporting Info Page 14

$1.21-1.32(\mathrm{~m}, 5 \mathrm{H}), 1.51(\mathrm{ddd}, J=7.2,10.8,18.0 \mathrm{~Hz}, 1 \mathrm{H}), 1.71(\mathrm{dt}, J=$ 18.0, $5.2 \mathrm{~Hz}, 1 \mathrm{H}), 1.86(\mathrm{~s}, 2 \mathrm{H}), 1.96(\mathrm{~d}, J=16.0 \mathrm{~Hz}, 1 \mathrm{H}), 2.25(\mathrm{~d}, J=$ $16.0 \mathrm{~Hz}, 1 \mathrm{H}), 3.26(\mathrm{~d}, J=11.2 \mathrm{~Hz}, 1 \mathrm{H}), 3.31(\mathrm{~d}, J=11.2 \mathrm{~Hz}, 1 \mathrm{H}), 3.34$ $(\mathrm{d}, J=11.2 \mathrm{~Hz}, 1 \mathrm{H}), 3.37(\mathrm{~d}, J=11.2 \mathrm{~Hz}, 1 \mathrm{H}) ;{ }^{13} \mathrm{C} \mathrm{NMR}(100 \mathrm{MHz}$, $\left.\mathrm{C}_{6} \mathrm{D}_{6}\right) \delta 15.2,17.0,19.5,22.4,24.3,24.8,25.4,33.9,37.5,39.7,44.3,46.2$, 69.7, 69.7, 97.8, 133.0, 134.4, 204.5; LRMS (EI) $m / z 290\left(\mathrm{M}^{+}\right), 275,260$, 232, 217, 202, 187; HRMS (EI) calcd for $\mathrm{C}_{18} \mathrm{H}_{26} \mathrm{O}_{3}$ 290.1882, found 290.1901; Anal. Calcd for $\mathrm{C}_{18} \mathrm{H}_{26} \mathrm{O}_{3}: \mathrm{C}, 74.45 ; \mathrm{H}, 9.02$. Found: $\mathrm{C}, 74.51 ; \mathrm{H}$, 9.01 .

6,6-Bisbenzyloxymethylnon-8-en-3-yn-2-one (1a)

IR (neat) $2207,1675,1095 \mathrm{~cm}^{-1} ;{ }^{1} \mathrm{H}$ NMR $\left(400 \mathrm{MHz}, \mathrm{CDCl}_{3}\right) \delta 2.24(\mathrm{~d}, J=$ $6.8 \mathrm{~Hz}, 2 \mathrm{H}), 2.25(\mathrm{~s}, 3 \mathrm{H}), 2.47(\mathrm{~s}, 2 \mathrm{H}), 3.38(\mathrm{~s}, 4 \mathrm{H}), 4.49(\mathrm{~s}, 4 \mathrm{H})$, 5.05-5.11 (m, $2 \mathrm{H}), 5.69-5.80(\mathrm{~m}, 1 \mathrm{H}), 7.25-7.34(\mathrm{~m}, 10 \mathrm{H}),{ }^{13} \mathrm{C}$ NMR $(100$ $\left.\mathrm{MHz}, \mathrm{CDCl}_{3}\right) \delta 23.1,32.9,36.7,42.6,71.6,73.3,83.1,91.4,118.4,127.2$ 127.3, 128.1, 133.0, 138.2, 184.1; LRMS (El) $m / z 376\left(\mathrm{M}^{+}\right), 129,107,91$, 
(C) 2005 American Chemical Society, Organometallics, Tanaka om058053i Supporting Info Page 15

43; HRMS (EI) calcd for $\mathrm{C}_{25} \mathrm{H}_{28} \mathrm{O}_{3}, 376.20383$, found 306.20414; Anal.

Calcd for $\mathrm{C}_{25} \mathrm{H}_{28} \mathrm{O}_{3}: \mathrm{C}, 79.75 ; \mathrm{H}, 7.50$. Found: $\mathrm{C}, 79.52 ; \mathrm{H}, 7.66$.

\section{5-(5-Allyl-2,2-dimethyl-[1,3]dioxan-5-yl)pent-3-yn-2-one (1b)}

IR (neat) $2209,1678,1639,1421 \mathrm{~cm}^{-1} ;{ }^{1} \mathrm{H}$ NMR $\left(270 \mathrm{MHz}, \mathrm{CDCl}_{3}\right) \delta 1.41$

(s, $3 \mathrm{H}), 1.42(\mathrm{~s}, 3 \mathrm{H}), 2.12(\mathrm{~d}, J=8.1 \mathrm{~Hz}, 2 \mathrm{H}), 2.34(\mathrm{~s}, 3 \mathrm{H}), 2.63(\mathrm{~s}, 2 \mathrm{H})$,

$3.61(\mathrm{~d}, J=11.9 \mathrm{~Hz}, 2 \mathrm{H}), 3.72(\mathrm{~d}, J=11.9 \mathrm{~Hz}, 2 \mathrm{H}), 5.12(\mathrm{~d}, J=2.7 \mathrm{~Hz}, 1$

$\mathrm{H}), 5.17(\mathrm{~s}, 1 \mathrm{H}), 5.72(\mathrm{dt}, J=2.7,8.1 \mathrm{~Hz}, 1 \mathrm{H}) ;{ }^{13} \mathrm{C} \mathrm{NMR}(100 \mathrm{MHz}$,

$\left.\mathrm{CDCl}_{3}\right) \delta 20.7,22.9,27.0,32.9,36.1,37.2,66.6,83.6,90.4,98.1,119.2$,

131.2, 183.8; LRMS (EI) $m / 2221\left(\mathrm{M}^{+}-\mathrm{CH}_{3}\right), 161,149,133,119,105,91$,

79, 57; HRMS (EI) calcd for $\mathrm{C}_{13} \mathrm{H}_{17} \mathrm{O}_{3}$ 221.1188, found 221.1183; Anal.

Calcd for $\mathrm{C}_{14} \mathrm{H}_{20} \mathrm{O}_{3}: \mathrm{C}, 71.16 ; \mathrm{H}$, 8.53. Found: $\mathrm{C}, 71.16 ; \mathrm{H}, 8.56$.

1-(5-Allyl-2,2-dimethyl-1,3-dioxan-5-yl)hept-2-yn-4-one (1c)

IR (neat) $2210,1674,1639 \mathrm{~cm}^{-1} ;{ }^{1} \mathrm{H}$ NMR $\left(400 \mathrm{MHz}, \mathrm{CDCl}_{3}\right) \delta 0.95(\mathrm{t}, J=$

$7.5 \mathrm{~Hz}, 3 \mathrm{H}), 1.41(\mathrm{~s}, 3 \mathrm{H}), 1.43(\mathrm{~s}, 3 \mathrm{H}), 1.70(\mathrm{tq}, J=7.6,7.6 \mathrm{~Hz}, 2 \mathrm{H})$, 
(C) 2005 American Chemical Society, Organometallics, Tanaka om058053i Supporting Info Page 16

$2.13(\mathrm{~d}, J=8.0 \mathrm{~Hz}, 2 \mathrm{H}), 2.52(\mathrm{t}, J=7.6 \mathrm{~Hz}, 2 \mathrm{H}), 2.63(\mathrm{~s}, 2 \mathrm{H}), 3.62(\mathrm{~d}, J$

$=12.0 \mathrm{~Hz}, 2 \mathrm{H}), 3.71(\mathrm{~d}, J=12.0 \mathrm{~Hz}, 2 \mathrm{H}), 5.14(\mathrm{~d}, J=18.0 \mathrm{~Hz}, 1 \mathrm{H}), 5.15$

(d, $J=10.4 \mathrm{~Hz}, 1 \mathrm{H}), 5.72(\mathrm{ddt}, J=10.4,18.0,8.0 \mathrm{~Hz}, 1 \mathrm{H}) ;{ }^{13} \mathrm{C}$ NMR $(100$

$\left.\mathrm{MHz}, \mathrm{CDCl}_{3}\right) \delta 13.7,17.8,20.8,23.0,27.0,36.1,37.2,47.5,66.7,83.2$,

90.4, 98.2, 119.3, 131.3, 187.4; LRMS (El) $\mathrm{m} / \mathrm{z} 264\left(\mathrm{M}^{+}\right), 249,221,105$,

91, 71, 43; HRMS (EI) calcd for $\mathrm{C}_{16} \mathrm{H}_{24} \mathrm{O}_{3}$ 264.1741, found 264.1733; Anal.

Calcd for $\mathrm{C}_{16} \mathrm{H}_{24} \mathrm{O}_{3}: \mathrm{C}, 72.69 ; \mathrm{H}, 9.15$. Found: $\mathrm{C}, 72.43 ; \mathrm{H}, 9.25$.

6-(5-Allyl-2,2-dimethyl-1,3-dioxan-5-yl)-2-methylhex-4-yn-3-one (1d)

IR (neat) 2209, 1673, $1640 \mathrm{~cm}^{-1} ;{ }^{1} \mathrm{H}$ NMR $\left(270 \mathrm{MHz}, \mathrm{CDCl}_{3}\right) \delta 1.19(\mathrm{~d}, J=$

$6.5 \mathrm{~Hz}, 6 \mathrm{H}), 1.41(\mathrm{~s}, 3 \mathrm{H}), 1.43(\mathrm{~s}, 3 \mathrm{H}), 2.15(\mathrm{~d}, J=7.6 \mathrm{~Hz}, 2 \mathrm{H})$,

2.58-2.68 (m, $1 \mathrm{H}), 3.63(\mathrm{~d}, J=11.9 \mathrm{~Hz}, 2 \mathrm{H}), 3.72(\mathrm{~d}, J=11.9 \mathrm{~Hz}, 2 \mathrm{H})$,

$5.15(\mathrm{~d}, J=11.6 \mathrm{~Hz}, 1 \mathrm{H}), 5.15(\mathrm{~d}, J=15.4 \mathrm{~Hz}, 1 \mathrm{H}), 5.73$ (ddt, $J=11.6$,

15.4, 7.6 Hz, $1 \mathrm{H}) ;{ }^{13} \mathrm{C}$ NMR $\left(100 \mathrm{MHz}, \mathrm{CDCl}_{3}\right) \delta 18.2,21.0,23.1,26.8$,

$36.1,37.2,43.2,66.7,82.2,91.3,98.2,119.3,131.3,191.4$; LRMS (EI) $\mathrm{m} / \mathrm{z}$

$263\left(\mathrm{M}^{+}-\mathrm{H}\right), 249,221,119,105,91,43$; HRMS (EI) calcd for $\mathrm{C}_{16} \mathrm{H}_{23} \mathrm{O}_{3}$ 
(C) 2005 American Chemical Society, Organometallics, Tanaka om058053i Supporting Info Page 17

263.16471, found 263.16378; Anal. Calcd for $\mathrm{C}_{16} \mathrm{H}_{24} \mathrm{O}_{3}$ : C, 72.69; H, 9.15.

Found: $\mathrm{C}, 72.74 ; \mathrm{H}, 9.17$.

4-(5-Allyl-2,2-dimethyl-1,3-dioxan-5-yl)-1-cyclohexylbut-2-yn-1-one

(1e)

IR (neat) $2209,1668,1640 \mathrm{~cm}^{-1} ;{ }^{1} \mathrm{H} \mathrm{NMR}\left(400 \mathrm{MHz}, \mathrm{CDCl}_{3}\right) \delta 1.16-1.47$

$(\mathrm{m}, 5 \mathrm{H}), 1.41(\mathrm{~s}, 3 \mathrm{H}), 1.43(\mathrm{~s}, 3 \mathrm{H}), 1.64-1.68(\mathrm{~m}, 1 \mathrm{H}), 1.76-1.80(\mathrm{~m}, 2$

H), 1.95-1.99 (m, $2 \mathrm{H}), 2.14(\mathrm{~d}, J=7.6 \mathrm{~Hz}, 2 \mathrm{H}), 2.33-2.41(\mathrm{~m}, 1 \mathrm{H}), 2.63$

(s, $2 \mathrm{H}), 3.63(\mathrm{~d}, J=12.0 \mathrm{~Hz}, 2 \mathrm{H}), 3.71(\mathrm{~d}, J=12.0 \mathrm{~Hz}, 2 \mathrm{H}), 5.15(\mathrm{~d}, J=$

$18.0 \mathrm{~Hz}, 1 \mathrm{H}), 5.15(\mathrm{~d}, J=9.2 \mathrm{~Hz}, 1 \mathrm{H}), 5.73(\mathrm{ddt}, J=9.2,18.0,7.6 \mathrm{~Hz}, 1$

$\mathrm{H}) ;{ }^{13} \mathrm{C}$ NMR $\left(100 \mathrm{MHz}, \mathrm{CDCl}_{3}\right) \delta 21.1,23.1,25.5,26.0,26.7,28.4,36.1$,

$37.2,52.3,66.7,82.5,91.0,98.2,119.3,131.3,190.7 ;$ LRMS (EI) $\mathrm{m} / 2304$

$\left(\mathrm{M}^{+}\right), 289,263,221,91,83,79,55$; HRMS (EI) calcd for $\mathrm{C}_{19} \mathrm{H}_{28} \mathrm{O}_{3}$

304.2033, found 304.2036; Anal. Calcd for $\mathrm{C}_{19} \mathrm{H}_{28} \mathrm{O}_{3}$ : C, 74.96; $\mathrm{H}, 9.27$.

Found: C, 74.86; H, 9.15. 
(C) 2005 American Chemical Society, Organometallics, Tanaka om058053i Supporting Info Page 18

\section{1-(5-Allyl-2,2-dimethyl-1,3-dioxan-5-yl)-7-phenylhept-2-yn-4-one (1f)}

IR (neat) 2209, 1673, $1118 \mathrm{~cm}^{-1}$; 'H NMR (400 MHz, $\left.\mathrm{CDCl}_{3}\right) \delta 1.40(\mathrm{~s}, 3$

H), $1.42(\mathrm{~s}, 3 \mathrm{H}), 2.00(\mathrm{tt}, J=7.6,7.6 \mathrm{~Hz}, 2 \mathrm{H}), 2.10(\mathrm{~d}, J=7.6 \mathrm{~Hz}, 2 \mathrm{H})$,

$2.56(\mathrm{t}, J=7.6 \mathrm{~Hz}, 2 \mathrm{H}), 2.63(\mathrm{~s}, 2 \mathrm{H}), 2.65(\mathrm{t}, J=7.6 \mathrm{~Hz}, 2 \mathrm{H}), 3.60(\mathrm{~d}, J=$

$12.0 \mathrm{~Hz}, 2 \mathrm{H}), 3.70(\mathrm{~d}, J=12.0 \mathrm{~Hz}, 2 \mathrm{H}), 5.12(\mathrm{~d}, J=5.2 \mathrm{~Hz}, 1 \mathrm{H}), 5.13(\mathrm{~d}$,

$J=16.4 \mathrm{~Hz}, 1 \mathrm{H}), 5.71(\mathrm{ddt}, J=5.2,16.4,7.6 \mathrm{~Hz}, 1 \mathrm{H}), 7.16-7.31(\mathrm{~m}, 5 \mathrm{H})$;

${ }^{13} \mathrm{C}$ NMR $\left(67.5 \mathrm{MHz}, \mathrm{CDCl}_{3}\right) \delta 20.6,22.8,25.6,26.8,34.8,35.9,37.1$, $44.8,66.7,83.2,90.7,98.3,119.5,126.0,128.4,128.4,131.5,141.2,187.4$

LRMS (EI) $m / z 340\left(\mathrm{M}^{+}\right), 325,299,267,252,117,104,91,43 ;$ HRMS (EI) calcd for $\mathrm{C}_{22} \mathrm{H}_{28} \mathrm{O}_{3} 340.2038$, found 340.2044; Anal. Calcd for $\mathrm{C}_{22} \mathrm{H}_{28} \mathrm{O}_{3}: \mathrm{C}$, 77.61; H, 8.29. Found: C, 77.72; H, 8.24.

\section{1-(5-Allyl-2,2-dimethyl-1,3-dioxan-5-yl)-7-benzyloxyhept-2-yn-4-one}

\section{(1g)}

IR (neat) 2209, 1674, 1639, $1117 \mathrm{~cm}^{-1} ;{ }^{1} \mathrm{H}$ NMR $\left(400 \mathrm{MHz}, \mathrm{CDCl}_{3}\right) \delta 1.41$

$(\mathrm{s}, 3 \mathrm{H}), 1.42(\mathrm{~s}, 3 \mathrm{H}), 1.97(\mathrm{tt}, J=6.4,7.6 \mathrm{~Hz}, 2 \mathrm{H}), 2.11(\mathrm{~d}, J=7.6 \mathrm{~Hz}, 2$ 
(C) 2005 American Chemical Society, Organometallics, Tanaka om058053i Supporting Info Page 19

H), $2.63(\mathrm{~s}, 2 \mathrm{H}), 2.69(\mathrm{t}, J=7.6 \mathrm{~Hz}, 2 \mathrm{H}), 3.50(\mathrm{t}, J=6.4 \mathrm{~Hz}, 2 \mathrm{H}), 3.61(\mathrm{~d}$, $J=12.0 \mathrm{~Hz}, 2 \mathrm{H}), 3.71(\mathrm{~d}, J=12.0 \mathrm{~Hz}, 2 \mathrm{H}), 4.49(\mathrm{~s}, 2 \mathrm{H}), 5.14(\mathrm{~d}, J=$ $11.2 \mathrm{~Hz}, 1 \mathrm{H}), 5.14(\mathrm{~d}, J=14.8 \mathrm{~Hz}, 1 \mathrm{H}), 5.71(\mathrm{ddt}, J=11.2,14.8,7.6 \mathrm{~Hz}$, $1 \mathrm{H})$, 7.26-7.37 (m, $5 \mathrm{H}) ;{ }^{13} \mathrm{C}$ NMR $\left(67.5 \mathrm{MHz}, \mathrm{CDCl}_{3}\right) \delta$ 20.6, 22.8, 24.1, $26.8,35.9,37.1,42.4,66.6,68.9,72.9,83.2,90.7,98.3,119.4,127.5,127.6$ 128.3, 131.5, 138.3, 187.2; LRMS (EI) $m / 2370\left(\mathrm{M}^{+}\right), 355,105,91,43$; HRMS (EI) calcd for $\mathrm{C}_{23} \mathrm{H}_{30} \mathrm{O}_{4} 370.2144$, found 370.2142; Anal. Calcd for $\mathrm{C}_{23} \mathrm{H}_{30} \mathrm{O}_{4}: \mathrm{C}, 74.54 ; \mathrm{H}, 8.22$. Found: $\mathrm{C}, 74.56 ; \mathrm{H}, 8.16$.

6-(5-Allyl-2,2-dimethyl-1,3-dioxan-5-yl)-2,2-dimethylhex-4-yn-3-one (1h)

IR (neat) 2208, 1670, $1639 \mathrm{~cm}^{-1} ;{ }^{1} \mathrm{H}$ NMR (400 MHz, $\left.\mathrm{CDCl}_{3}\right) \delta 1.20$ (s, 9 H), $1.41(\mathrm{~s}, 3 \mathrm{H}), 1.43(\mathrm{~s}, 3 \mathrm{H}), 2.16(\mathrm{~d}, J=7.6 \mathrm{~Hz}, 2 \mathrm{H}), 2.64(\mathrm{~s}, 2 \mathrm{H}), 3.63$ $(\mathrm{d}, J=12.0 \mathrm{~Hz}, 2 \mathrm{H}), 3.72(\mathrm{~d}, J=12.0 \mathrm{~Hz}, 2 \mathrm{H}), 5.15(\mathrm{~d}, J=9.6 \mathrm{~Hz}, 1 \mathrm{H})$, $5.15(\mathrm{~d}, J=17.6 \mathrm{~Hz}, 1 \mathrm{H}), 5.73(\mathrm{ddt}, J=9.6,17.6,7.6 \mathrm{~Hz}, 1 \mathrm{H}) ;{ }^{13} \mathrm{C} \mathrm{NMR}$ $\left(100 \mathrm{MHz}, \mathrm{CDCl}_{3}\right) \delta 21.2,23.1,26.3,26.6,36.2,37.2,44.7,66.8,81.2$, 
(C) 2005 American Chemical Society, Organometallics, Tanaka om058053i Supporting Info Page 20

91.8, 98.2, 119.3, 131.4, 193.4; LRMS (EI) $\mathrm{m} / \mathrm{z} 278\left(\mathrm{M}^{+}\right), 263,190,175$,

91, 79, 57, 43; HRMS (EI) calcd for $\mathrm{C}_{17} \mathrm{H}_{26} \mathrm{O}_{3}$ 278.1878, found 278.1880;

Anal. Calcd for $\mathrm{C}_{17} \mathrm{H}_{26} \mathrm{O}_{3}: \mathrm{C}, 73.34 ; \mathrm{H}, 9.41$. Found: $\mathrm{C}, 73.26 ; \mathrm{H}, 9.42$.

$N$-Allyl-4-methyl- $N$-(4-oxopent-2-ynyl)benzenesulfonamide (1i)

Mp. $39-43^{\circ} \mathrm{C}$; IR (neat) $2210,1680,1352,1164 \mathrm{~cm}^{-1}$; ${ }^{1} \mathrm{H}$ NMR $(400 \mathrm{MHz}$,

$\left.\mathrm{CDCl}_{3}\right) \delta 2.10(\mathrm{~s}, 3 \mathrm{H}), 2.43(\mathrm{~s}, 3 \mathrm{H}), 3.83(\mathrm{~d}, J=6.4 \mathrm{~Hz}, 2 \mathrm{H}), 4.24(\mathrm{~s}, 2 \mathrm{H})$,

5.27 (dd, $J=1.2,3.2 \mathrm{~Hz}, 1 \mathrm{H}), 5.30$ (dd, $J=1.2,11.6 \mathrm{~Hz}, 2 \mathrm{H}$ ), 5.73 (ddt, $J$

$=3.2,11.6,6.4 \mathrm{~Hz}, 1 \mathrm{H}), 7.32(\mathrm{~d}, J=8.0 \mathrm{~Hz}, 2 \mathrm{H}), 7.74(\mathrm{~d}, J=8.0 \mathrm{~Hz}, 2$

$\mathrm{H}) ;{ }^{13} \mathrm{C}$ NMR $\left(100 \mathrm{MHz}, \mathrm{CDCl}_{3}\right) \delta 21.7,32.4,36.0,49.7,84.1,84.6,120.3$,

127.5, 129.5, 131.3, 135.3, 143.8, 182.7; LRMS (EI) $\mathrm{m} / \mathrm{z} 291\left(\mathrm{M}^{+}\right), 210$

155, 136, 91; HRMS (EI) calcd for $\mathrm{C}_{15} \mathrm{H}_{17} \mathrm{O}_{3} \mathrm{NS} 291.0929$, found 291.0927.

\section{5-Allyloxypent-3-yn-2-one (1j)}

IR (neat) 2208, 1682, $1093 \mathrm{~cm}^{-1} ;{ }^{1} \mathrm{H}$ NMR $\left(400 \mathrm{MHz}, \mathrm{CDCl}_{3}\right) \delta 2.37(\mathrm{~s}, 3$

H), 4.08 (ddd, $J=1.2,1.2,5.6 \mathrm{~Hz}, 2 \mathrm{H}$ ), $4.32(\mathrm{~s}, 2 \mathrm{H}), 5.26$ (dd, $J=1.2$, 
(C) 2005 American Chemical Society, Organometallics, Tanaka om058053i Supporting Info Page 21

$10.4 \mathrm{~Hz}, 1 \mathrm{H}), 5.33(\mathrm{dd}, J=1.2,17.6 \mathrm{~Hz}, 1 \mathrm{H}), 5.90(\mathrm{ddt}, J=10.4,17.6,5.6$

$\mathrm{Hz}, 1 \mathrm{H}) ;{ }^{13} \mathrm{C}$ NMR $\left(100 \mathrm{MHz}, \mathrm{CDCl}_{3}\right) \delta 32.7,57.0,71.1,85.6,87.3,118.3$,

133.2, 183.5; LRMS (EI) $m / 2123\left(\mathrm{M}^{+}-\mathrm{CH}_{3}\right), 109,95,82,58,43$; HRMS

(EI) calcd for $\mathrm{C}_{7} \mathrm{H}_{7} \mathrm{O}_{2}$ 123.0444, found 123.0445; Anal. Calcd for $\mathrm{C}_{8} \mathrm{H}_{10} \mathrm{O}_{2}$ :

C, 69.54; H, 7.30. Found: C, 69.38; H, 7.10.

\section{4-(5-Allyl-2,2-dimethyl-[1,3]dioxan-5-yl)but-2-ynal (1k)}

IR (neat) 2198, $1667 \mathrm{~cm}^{-1}$; ${ }^{1} \mathrm{H}$ NMR (400 MHz, $\left.\mathrm{CDCl}_{3}\right) \delta 1.41(\mathrm{~s}, 3 \mathrm{H})$,

$1.43(\mathrm{~s}, 3 \mathrm{H}), 2.11(\mathrm{~d}, J=7.6 \mathrm{~Hz}, 2 \mathrm{H}), 2.71(\mathrm{~s}, 2 \mathrm{H}), 3.61(\mathrm{~d}, J=12.0 \mathrm{~Hz}, 2$

H), $3.73(\mathrm{~d}, J=12.0 \mathrm{~Hz}, 2 \mathrm{H}), 5.14(\mathrm{~d}, J=4.4 \mathrm{~Hz}, 1 \mathrm{H}), 5.17(\mathrm{~s}, 1 \mathrm{H}), 5.71$

(dt, $J=4.4,7.6 \mathrm{~Hz}, 1 \mathrm{H}), 9.20(\mathrm{~s}, 1 \mathrm{H}) ;{ }^{13} \mathrm{C}$ NMR $\left(100 \mathrm{MHz}, \mathrm{CDCl}_{3}\right) \delta 20.3$,

$23.1,27.4,36.2,37.1,66.6,83.8,95.7,98.2,119.5,131.0,176.4$; LRMS

(EI) $m / z 207\left(\mathrm{M}^{+}-\mathrm{CH}_{3}\right), 149,105,91,43$; HRMS (EI) calcd for $\mathrm{C}_{12} \mathrm{H}_{15} \mathrm{O}_{3}$

207.1033, found 207.1027; Anal. Calcd for $\mathrm{C}_{13} \mathrm{H}_{18} \mathrm{O}_{3}$ : C, 70.24; $\mathrm{H}, 8.16$.

Found: C, 70.34; $\mathrm{H}, 8.31$. 
(C) 2005 American Chemical Society, Organometallics, Tanaka om058053i Supporting Info Page 22

\section{2d-6-(5-Allyl-2,2-dimethyl-1,3-dioxan-5-yl)-2-methylhex-4-yn-3-one}

IR (neat) 2208, $1671 \mathrm{~cm}^{-1} ;{ }^{1} \mathrm{H}$ NMR $\left(270 \mathrm{MHz}, \mathrm{CDCl}_{3}\right) \delta 1.18(\mathrm{~s}, 6 \mathrm{H})$,

$1.41(\mathrm{~s}, 3 \mathrm{H}), 1.43(\mathrm{~s}, 3 \mathrm{H}), 2.14(\mathrm{~d}, J=7.6 \mathrm{~Hz}, 2 \mathrm{H}), 2.64(\mathrm{~s}, 2 \mathrm{H}), 3.62(\mathrm{~d}$, $J=11.3 \mathrm{~Hz}, 2 \mathrm{H}), 3.72(\mathrm{~d}, J=11.3 \mathrm{~Hz}, 2 \mathrm{H}), 5.14(\mathrm{~d}, J=10.8 \mathrm{~Hz}, 1 \mathrm{H})$,

$5.15(\mathrm{~d}, J=5.9 \mathrm{~Hz}, 1 \mathrm{H}), 5.72(\mathrm{ddt}, J=5.9,10.8,7.6 \mathrm{~Hz}, 1 \mathrm{H}) ;{ }^{13} \mathrm{C}$ NMR $\left(67.5 \mathrm{MHz}, \mathrm{CDCl}_{3}\right) \delta 17.9(\mathrm{~s}), 20.8(\mathrm{~s}), 22.8(\mathrm{~s}), 26.6(\mathrm{~s}), 35.9(\mathrm{~s}), 37.1(\mathrm{~s})$, $42.6(\mathrm{t}), 66.7(\mathrm{~s}), 82.1(\mathrm{~s}), 91.3(\mathrm{~s}), 98.3(\mathrm{~s}), 119.4(\mathrm{~s}), 131.5(\mathrm{~s}), 191.9(\mathrm{~s})$; LRMS (EI) m/z $265\left(\mathrm{M}^{+}\right), 249,221,105,91,79,71,43 ;$ HRMS (EI) calcd for $\mathrm{C}_{16} \mathrm{H}_{23}{ }^{2} \mathrm{HO}_{3} 265.1787$, found 265.1786 .

\section{5-[5-(2d)-Allyl-2,2-dimethyl-[1,3]dioxan-5-yl]pent-3-yn-2-one (1b-D)}

IR (neat) 2209, 1677, 1623, $1421 \mathrm{~cm}^{-1} ;{ }^{1} \mathrm{H}$ NMR $\left(270 \mathrm{MHz}, \mathrm{CDCl}_{3}\right) \delta 1.40$ (s, $3 \mathrm{H}), 1.43$ (s, $3 \mathrm{H}), 2.11(\mathrm{~s}, 2 \mathrm{H}), 2.34(\mathrm{~s}, 3 \mathrm{H}), 2.63$ (s, $2 \mathrm{H}), 3.61$ (d, $J=$ $12.2 \mathrm{~Hz}, 2 \mathrm{H}), 3.71(\mathrm{~d}, J=12.2 \mathrm{~Hz}, 2 \mathrm{H}), 5.15$ (brs, $2 \mathrm{H}) ;{ }^{13} \mathrm{C}$ NMR $(67.5$ $\left.\mathrm{MHz}, \mathrm{CDCl}_{3}\right) \delta 20.4(\mathrm{~s}), 22.7(\mathrm{~s}), 26.8(\mathrm{~s}), 32.7(\mathrm{~s}), 35.9(\mathrm{~s}), 36.9(\mathrm{~s}), 66.6$ 
(C) 2005 American Chemical Society, Organometallics, Tanaka om058053i Supporting Info Page 23

$(\mathrm{s}), 83.6(\mathrm{~s}), 90.4(\mathrm{~s}), 98.2(\mathrm{~s}), 119.3(\mathrm{~s}), 131.1(\mathrm{t}), 184.2(\mathrm{~s}):$

$m / z 237\left(\mathrm{M}^{+}\right), 222,162,149,134,120,106,92,80 ; \mathrm{HRMS}$

$\mathrm{C}_{14} \mathrm{H}_{19}{ }^{2} \mathrm{HO}_{3} 237.1474$, found 237.1470 .

\section{1-(5-Allyl-2,2-dimethyl-1,3-dioxan-5-yl)non-8-en-2-yn-4-oli}

IR (neat) $2210,1674,1640,1119 \mathrm{~cm}^{-1}$; ${ }^{1} \mathrm{H}$ NMR $(270 \mathrm{MHz}$,

$(\mathrm{s}, 3 \mathrm{H}), 1.43(\mathrm{~s}, 3 \mathrm{H}), 1.77(\mathrm{t}, J=7.3 \mathrm{~Hz}, 2 \mathrm{H}), 2.05-2.14(\mathrm{~m}$,

$J=7.3 \mathrm{~Hz}, 2 \mathrm{H}), 2.63(\mathrm{~s}, 2 \mathrm{H}), 3.61(\mathrm{~d}, J=12.2 \mathrm{~Hz}, 2 \mathrm{H}), 3$.

$\mathrm{Hz}, 2 \mathrm{H}), 4.97-5.17(\mathrm{~m}, 4 \mathrm{H}), 5.645 .85(\mathrm{~m}, 2 \mathrm{H}) ;{ }^{13} \mathrm{C} \mathrm{NM}$

$\left.\mathrm{CDCl}_{3}\right) \delta 20.6,22.8,23.1,26.8,32.8,36.0,37.1,44.8,66$.

98.3, 115.5, 119.5, 131.5, 137.6, 187.6; LRMS (EI) $m / z 290$

249, 59, 43; HRMS (EI) calcd for $\mathrm{C}_{18} \mathrm{H}_{26} \mathrm{O}_{3}$ 290.1882, fot:

Anal. Calcd for $\mathrm{C}_{18} \mathrm{H}_{26} \mathrm{O}_{3}: \mathrm{C}, 74.45 ; \mathrm{H}, 9.02$. Found: $\mathrm{C}, 74.29$; 